\title{
Effect of substrate misorientation on the InAs/InAIAs/InP nanostructure morphology and lateral composition modulation in the InAIAs matrix
}

\author{
W. Lei $^{\text {a) }}$ \\ Key Laboratory of Semiconductor Materials Science, Institute of Semiconductors, \\ Chinese Academy of Sciences, P.O. Box 912, Beijing, 100083, People's Republic of China \\ and Department of Physics, Universität Duisburg-Essen, 47048, Lotharstr. 1, Duisburg, Germany \\ Y. L. Wang, ${ }^{\text {b) }}$ Y. H. Chen, P. Jin, X. L. Ye, B. Xu, and Z. G. Wang \\ Key Laboratory of Semiconductor Materials Science, Institute of Semiconductors, \\ Chinese Academy of Sciences, P.O. Box 912, Beijing 100083, People's Republic of China
}

(Received 27 November 2006; accepted 2 January 2007; published online 9 March 2007)

\begin{abstract}
The authors report the self-organized growth of InAs/InAlAs quantum wires on nominal (001) InP substrate and (001) InP substrates misoriented by $2^{\circ}, 4^{\circ}$, and $8^{\circ}$ towards both $[-110]$ and [110]. The influence of substrate misorientation on the structural and optical properties of these InAs/InAlAs quantum wires is studied by transmission electron microscopy and photoluminescence measurements. Compared with that grown on nominal (001) InP substrate, the density of InAs/InAlAs quantum wires grown on misoriented $\mathrm{InP}(001)$ substrates is enhanced. A strong lateral composition modulation effect take place in the InAlAs buffer layers grown on misoriented InP substrates with large off-cut angles $\left(4^{\circ}\right.$ and $\left.8^{\circ}\right)$, which induces a nucleation template for the first-period InAs quantum wires and greatly improve the size distribution of InAs quantum wires. InAs/InAlAs quantum wires grown on $\operatorname{InP}(001)$ substrate $8^{\circ}$ off cut towards [-110] show the best size homogeneity and photoluminescence intensity. (C) 2007 American Institute of Physics.
\end{abstract} [DOI: $10.1063 / 1.2711778]$

Recently, much effort has been devoted to the growth of self-organized nanostructures [quantum dots (QDs) and quantum wires (QWRs)] due to their potential applications in devices. ${ }^{1-5}$ A key point for their device applications is to grow these nanostructures with high density and homogeneous size distribution, which presents a challenge because of the random nature of self-assembled growth process. One way is to grow nanostructure superlattices. ${ }^{6,7}$ Another way is to grow nanostructures on misoriented substrates. ${ }^{8-11}$ Due to the preferential nucleation sites along the surface steps provided by misoriented substrates, nanostructures with high density have been realized on misoriented substrates for InAs/InP, InP/GaAs, and InAs/GaAs QD systems. ${ }^{8-11}$

As to InAs/InAlAs/InP nanostructure system, the growth control is even more difficult partially due to the phase separation effect in InAlAs layers and the relatively weak driving force for island formation associated with low misfit strain $(3.2 \%){ }^{12,13}$ Though some effort has been devoted to the growth of these InAs/InAlAs/InP nanostructures, ${ }^{12-16}$ the growth quality and optical properties of these nanostructures are still not satisfying. In the meantime, most of the work about InAs/InAlAs nanostructures is focused on the growth of InAs nanostructures on nominal (001) InP substrates, ${ }^{12-16}$ little attention has been devoted to the growth of InAs islands on misoriented (001) InP substrates. ${ }^{17}$ In this work, we present a detailed study on the growth of InAs/InAlAs nanostructures on misoriented (001) InP substrates off cut towards both [-110] and [110] with different off-cut angles.

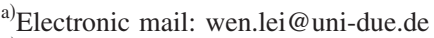

${ }^{b}$ Present address: R\&D Department in Beijing Tongmei Xtal Technology Co. Ltd., Tongzhou, Beijing 101113, People's Republic of China.
}

The structure and growth process of the samples were the same as those in Ref. 17. To ensure the same growth parameters, all substrates were soldered side by side with indium on a molybdenum holder. The structural and optical properties of the samples were studied by transmission electron microscopy (TEM) measurement with $g=002$ and photoluminescence (PL) measurements, respectively, the operation of which were also the same as those of Ref. 17.

The $g=002$ cross-sectional dark-field (DF) TEM images of the samples grown on nominal (001) InP substrate and (001) InP substrates off cut towards [-110] are shown in Fig. 1. Obviously, InAs/InAlAs QWRs oriented along the [1-10] direction are formed in the four samples, the shape of which is quite different from that of the InAs/InP nanostructures (QDs) grown on (001) InP substrate off cut towards $[-110]{ }^{8,10}$ The reasons for this shape difference will be discussed later. As shown in Fig. 1, the InAs QWR density is quite different depending on the substrate off-cut angles. The average densities (for six layers) are 255, 310, 300, and $285 \mu \mathrm{m}$ for the InAs QWRs grown on nominal (001) InP substrate and (001) InP substrates off cut by $2^{\circ}, 4^{\circ}$, and $8^{\circ}$ towards $[-110]$, respectively. The average densities of InAs QWRs grown on misoriented (001) InP substrates are all higher than that grown on nominal (001) InP substrate, which may be due to the increased number of the surface steps induced by misoriented (001) InP substrates. ${ }^{9,11,18}$

For (001) InP substrates, different off-cut directions with different off-cut angles can induce different types of surface steps with different densities. An off-cut towards the $[-110]([110])$ direction will lead to the surface steps running along the $[110]([-110])$ direction. In addition, the average step widths are 8.4, 4.2, and $2.1 \mathrm{~nm}$ for InP substrates off cut by $2^{\circ}, 4^{\circ}$, and $8^{\circ}$ towards both [-110] and [110], respectively. ${ }^{18}$ So, with the increasing substrate off-cut angle, 


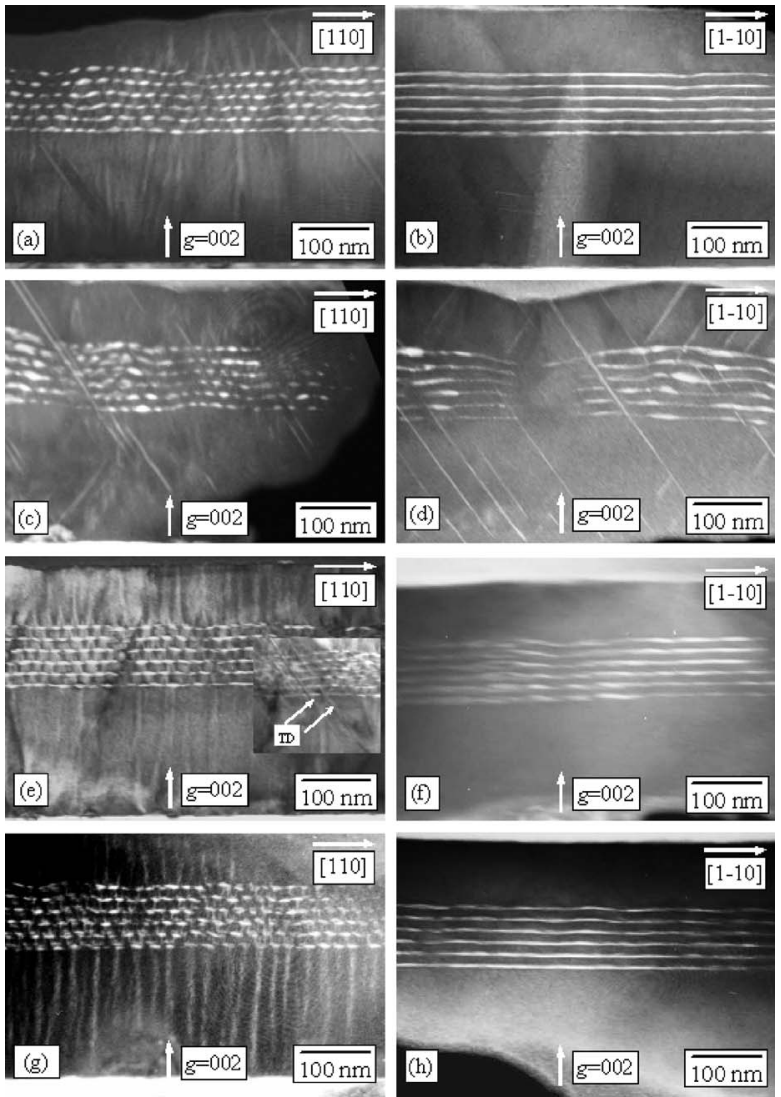

FIG. 1. $g=002$ cross-sectional dark-field TEM images of the samples grown on nominal InP (001) substrate [(a) and (b)] and misoriented InP (001) substrates off cut by $2^{\circ}\left[(\mathrm{c})\right.$ and (d)], $4^{\circ}[(\mathrm{e})$ and (f) $]$, and $8^{\circ}[(\mathrm{g})$ and $(\mathrm{h})]$ towards $[-110]$. Inset of (e) shows the threading dislocations existing in the sample.

the surface step number will increase and provide more nucleation sites for InAs nanostructures, leading to higher nanostructure density. ${ }^{9,11,18}$ However, as shown in Fig. 1, the InAs QWR density decreases when the off-cut angle is larger than $2^{\circ}$, which cannot be explained by the increased number of the surface steps. In previous works, ${ }^{13,14,17}$ lateral composition modulation (LCM) in InAlAs layers is reported to serve as a nucleation template for the growth of InAs QWRs and QDs, which suggests that LCM in InAlAs buffer layer will also influence the nucleation of InAs nanostructures (i.e., nanostructure density). From Figs. 1(a), 1(c), 1(e), and $1(\mathrm{~g})$, one can observe the alternate bright/dark contrast regions in InAlAs buffer layers which reveal a LCM along the [110] horizontal direction, where the brighter regions are Inrich and the darker regions Al-rich. ${ }^{14,17}$ The origin of this LCM in InAlAs buffer layers has been explained by Wang et al. with the bunching of surface steps. ${ }^{17}$ As shown in Fig. 1(c), the LCM effect in the InAlAs buffer layer grown on $2^{\circ}$ off-cut InP substrate is even weaker than that grown on nominal (001) InP substrate. The influence of LCM in such case is weak and can be neglected. The increased number of the surface steps induced by the $2^{\circ}$ off-cut substrate will provide much more nucleation sites for InAs QWRs, resulting in the highest QWR density. ${ }^{11}$ When the off-cut angle increases to $4^{\circ}$ and $8^{\circ}$, the LCM in InAlAs buffer layers becomes stronger. Especially, the LCM in the InAlAs buffer layer grown on $8^{\circ}$ off-cut InP substrate develops a well alternate In-rich and Al-rich regions. In this case, the LCM in InAlAs buffer layers will play a determinant role in the InAs Downloaded 08 Mar 2010 to 150.203 .243 .38 . Redistribution subject
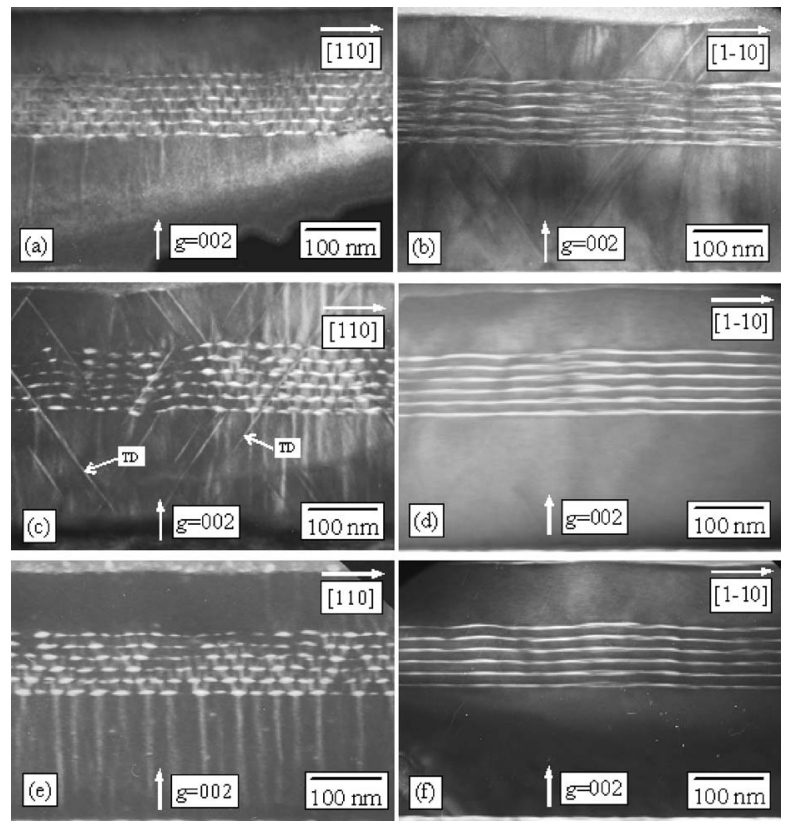

FIG. 2. $g=002$ cross-sectional dark-field TEM images of the samples grown on misoriented InP (001) substrates off cut by $2^{\circ}\left[(\mathrm{a})\right.$ and (b)], $4^{\circ}[(\mathrm{c})$ and (d)], and $8^{\circ}[(\mathrm{e})$ and (f)] towards [110]. The arrows in (c) denote the threading dislocations existing in the sample.

QWR nucleation process, and the In-rich regions in InAlAs buffer layers will provide preferential nucleation sites for InAs QWRs. ${ }^{14,17}$ So, the average lateral period (i.e., density) of the first-period InAs QWRs is mainly determined by the lateral period of the In-rich regions in InAlAs buffer layers $\left(\sim 18,20\right.$, and $22 \mathrm{~nm}$ for $2^{\circ}, 4^{\circ}$, and $8^{\circ}$ off-cut substrates here). ${ }^{14,17}$ As shown by Wang et al., ${ }^{17}$ the lateral period of the In-rich regions in InAlAs buffer layers is much larger than the distance between individual surface steps induced by offcutting, leading to the decreased density for InAs QWRs grown on $4^{\circ}$ and $8^{\circ}$ off-cut InP substrates. The smaller lateral period for the In-rich regions in InAlAs buffer layer grown on $4^{\circ}$ off-cut InP substrate (relative to that on $8^{\circ}$ off-cut InP substrate) may be related to the formation of thread dislocations (TDs) in the epilayers of the sample [as shown in the inset in Fig. 1(e) $].{ }^{17}$ The formation of TD will relax the strain and weaken the LCM effect in the $\mathrm{In}_{0.5} \mathrm{Al}_{0.5} \mathrm{As}$ layer.

Figure 2 shows the $g=002$ cross-sectional DF TEM images of the samples grown on (001) InP substrates off cut by $2^{\circ}, 4^{\circ}$, and $8^{\circ}$ towards [110]. InAs/InAlAs QWRs aligned along the [1-10] direction are also obtained in these three samples. Again, the samples demonstrate different InAs QWR densities for different off-cut angles, where the average densities (for six layers) are 255, 292, 265, and 268/ $\mu \mathrm{m}$ for the InAs QWRs grown on $0^{\circ}, 2^{\circ}, 4^{\circ}$, and $8^{\circ}$ off-cut substrates, respectively. This change of QWR density with increasing off-cut angle shows a similar trend to that grown on InP (001) substrates off cut towards [-110], which can also be explained by the competition between the increased number of surface steps and the LCM effect in InAlAs buffer layers. However, the densities of InAs QWRs grown on InP substrates off cut towards [110] are all lower than those grown on InP substrates off cut towards [-110] at each offcut angle, which may be due to the different surface terrace characteristics for the different off orientations (i.e., the extending direction of surface terrace is parallel (perpendicular) to AIP license or copyright; see http://apl.aip.org/apl/copyright.jsp 

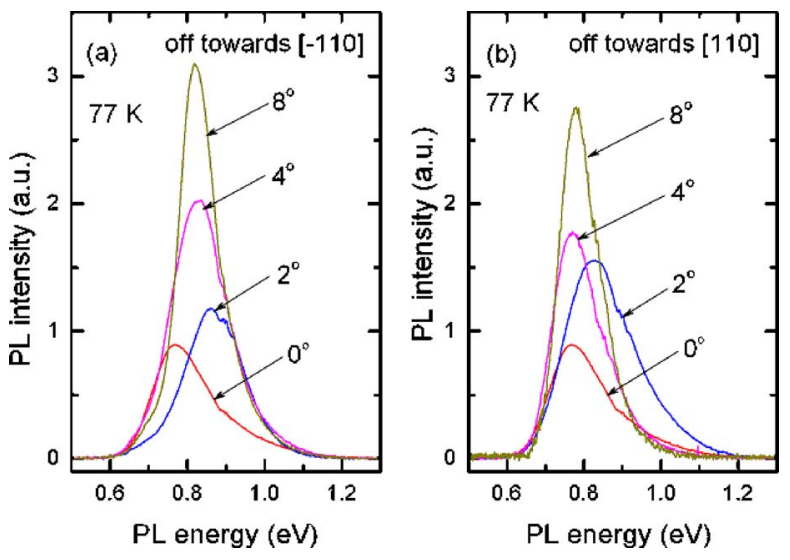

FIG. 3. (Color online) $77 \mathrm{~K}$ PL spectra of the samples grown on nominal InP (001) substrate and misoriented InP (001) substrates off cut towards $[-110]$ (a) and [110] (b)

to the [1-10] direction for InP substrates off cut towards [110] ([-110]). As shown in Figs. 1 and 2, no matter what the off-cut direction of substrates is, the LCM in the InAlAs buffer layer only forms along the [110] direction. In addition, the In-rich regions, which extend along the [1-10] direction, will provide the preferential nucleation sites for the firstperiod InAs islands, leading to the formation of InAs QWRs in the samples even for InP substrates off cut towards [-110].

PL spectra of the samples measured at $77 \mathrm{~K}$ are shown in Fig. 3. The PL peak is centered at $0.77,0.86,0.83$, and $0.82 \mathrm{eV}$ for InAs QWRs grown on nominal (001) InP substrate and InP substrates off cut by $2^{\circ}, 4^{\circ}$, and $8^{\circ}$ towards $[-110]$, respectively. And, the full widths at half maximum (FWHMs) of the PL spectra are 173, 176, 171, and $113 \mathrm{meV}$ for $0^{\circ}, 2^{\circ}, 4^{\circ}$, and $8^{\circ}$ off-cut samples, respectively. The small PL FWHM of InAs QWRs grown on $8^{\circ}$ off-cut InP substrate indicates a homogeneous size distribution for the InAs QWRs, which can be attributed to the strong LCM effect in InAlAs buffer layer. As shown in Fig. 1(g), well alternate In-rich and Al-rich regions are developed in the InAlAs buffer layer, and the In-rich regions will provide preferential nucleation sites for the first-period InAs QWRs, which greatly reduces the random nature of Stranski-Krastanov growth model and leads to a very homogeneous size distribution for InAs QWRs. Figure 3(b) shows the 77 K PL spectra of the samples grown on nominal (001) InP substrate and InP substrates off cut towards [110]. The PL peak is centered at $0.77,0.825,0.773$, and $0.78 \mathrm{eV}$ for InAs QWRs grown on the $0^{\circ}, 2^{\circ}, 4^{\circ}$, and $8^{\circ}$ off-cut substrates, respectively. In addition, the PL FWHMs are 173, 208, 149, and $120 \mathrm{meV}$ for the $0^{\circ}, 2^{\circ}, 4^{\circ}$, and $8^{\circ}$ off-cut samples, respectively. The decreased PL FWHMs of InAs QWRs grown on the $4^{\circ}$ and $8^{\circ}$ off-cut substrates can also be explained by the role of nucleation template provided by the LCM effect in InAlAs buffer layers. Compared with that of InAs QWRs grown on nominal (001) InP substrate, the PL intensities of InAs QWRs grown on misoriented InP substrates are all improved. The
InAs QWRs grown on InP substrate $8^{\circ}$ off cut towards $[-110]$ show the strongest PL spectrum [more than three times that grown on nominal (001) InP substrate], indicating a high growth quality.

In conclusion, the growth and optical properties of InAs/InAlAs QWRs on misoriented (001) InP substrates have been studied. Misoriented $\operatorname{InP}(001)$ substrates with small off-cut angles are helpful for the enhancement of InAs QWRs density, but are not good for the improvement of size distribution and optical properties of the nanostructures. Misoriented $\operatorname{InP}(001)$ substrates with high off-cut angles $\left(4^{\circ}\right.$ and $8^{\circ}$ ) are very useful for the enhancement of size homogeneity due to the strong LCM effect in the InAlAs buffer layers. InAs QWRs grown on (001) InP substrate $8^{\circ}$ off-cut towards [-110] demonstrates the best size homogeneity and PL efficiency, indicating that InP substrate $8^{\circ}$ off-cut towards $[-110]$ can be used to achieve InAs/InAlAs QWRs with high quality.

The work was supported by 973 program (No. 2006CB604908), National Natural Science Foundation of China (Nos. 60625402, 60390074, and 60576062), and an 863 program (2006AA03Z0408).

${ }^{1}$ For a review, see D. Bimberg, M. Grundmann, and N. N. Ledentsov, Quantum Dot Heterostructures (Wiley, New York, 1999).

${ }^{2}$ J. S. Kim, J. H. Lee, S. U. Hong, W. S. Han, H.-S. Kwack, C. W. Lee, and D. K. Oh, Appl. Phys. Lett. 85, 1033 (2004).

${ }^{3}$ F. Fossard, F. H. Julien, E. Péronne, A. Alexandrou, J. Brault, and M. Gendry, Infrared Phys. Technol. 42, 443 (2001).

${ }^{4}$ E. Finkman, S. Maimon, V. Immer, G. Bahir, S. E. Schacham, F. Fossard, F. H. Julien, J. Brault, and M. Gendry, Phys. Rev. B 63, 045323 (2001).

${ }^{5}$ E. Dupuy, P. Regreny, Y. Robach, M. Gendry, N. Chauvin, E. Tranvouez,

G. Bremond, C. Bru-Chevallier, and G. Patriarche, Appl. Phys. Lett. 89, 123112 (2006).

${ }^{6}$ Q. Xie, A. Madhukar, P. Chen, and N. P. Kobayashi, Phys. Rev. Lett. 75, 2542 (1995).

${ }^{7}$ J. Tersoff, C. Teichert, and M. G. Lagally, Phys. Rev. Lett. 76, 1675 (1996).

${ }^{8}$ O. Bierwagen and W. T. Masselink, Appl. Phys. Lett. 86, 113110 (2005).

${ }^{9}$ X. B. Zhang, R. D. Heller, M. S. Noh, R. D. Dupuis, G. Walter, and N. Holonyak, Jr., Appl. Phys. Lett. 83, 476 (2003).

${ }^{10}$ B. Salem, J. Olivares, G. Guillot, G. Bremond, J. Brault, C. Monat, M. Gendry, G. Hollinger, F. Hassen, and H. Maaref, Appl. Phys. Lett. 79, 4435 (2001).

${ }^{11}$ V. P. Evtikhiev, A. M. Boiko, I. V. Kudryashov, A. K. Kryganovskii, R. A. Suris, A. N. Titkov, and V. E. Tokranov, Semicond. Sci. Technol. 17, 545 (2002).

${ }^{12}$ F. A. Zhao, Y. H. Chen, X. L. Ye, P. Jin, B. Xu, Z. G. Wang, and C. L. Zhang, J. Phys.: Condens. Matter 16, 7603 (2004).

${ }^{13}$ J. Brault, M. Gendry, O. Marty, M. Pitaval, J. Olvares, G. Grenet, and G. Hollinger, Appl. Surf. Sci. 162/163, 584 (2000).

${ }^{14}$ W. Lei, Y. H. Chen, P. Jin, X. L. Ye, Y. L. Wang, B. Xu, and Z. G. Wang, Appl. Phys. Lett. 88, 063114 (2006).

${ }^{15}$ J. Brault, M. Gendry, G. Grenet, G. Hollinger, J. Olivares, B. Salem, T. Benyattou, and G. Bremond, J. Appl. Phys. 92, 506 (2002).

${ }^{16}$ B. H. Koo, T. Hanada, H. Makino, and T. Yao, Appl. Phys. Lett. 79, 4331 (2001).

${ }^{17}$ Y. L. Wang, P. Jin, X. L. Ye, C. L. Zhang, G. X. Shi, R. Y. Li, Y. H. Chen, and Z. G. Wang, Appl. Phys. Lett. 88, 123104 (2006).

${ }^{18}$ Zh. M. Wang, Sh. Seydmohamadi, J. H. Lee, and G. J. Salamo, Appl. Phys. Lett. 85, 5031 (2004). 